\title{
Synthesis of Imidazole-Based AIEgens with Wide Color Tunability and Exploration of their Biological Applications
}

\author{
Zhegang Song, Weijie Zhang, Meijuan Jiang, Herman H. Y. Sung, Ryan T. K. Kwok, \\ Han Nie, Ian D. Williams, Bin Liu,* and Ben Zhong Tang**
}

Research on aggregation-induced emission (AIE) has become increasingly popular recently and various AIE luminogens (AIEgens) have been developed based on tetraphenylethene, hexaphenylsilole, distyrylanthracene, tetraphenylpyrazine, etc. However, facile tuning of the AlEgen emissions in a wide range remains challenging. Herein, a novel series of AIEgens is reported, based on imidazole-cored molecular rotors, with facile synthesis and emission colors covering the whole visible spectrum. Moreover, these imidazole derivatives exhibit biological functions unique among the AIEgens, including mitochondria-specific imaging and antifungal activity. Benefiting from the easy preparation and the tunable emission, the imidazole derivatives are expected to not only diversify the family of AIEgens but also enrich their biological applications.

\section{Introduction}

Since the concept of aggregation-induced emission (AIE) was coined in 2001, luminogens with AIE characteristics (AIEgens) have undergone a rapid expansion in the diversity of molecules, mechanistic understanding, and applications. ${ }^{[1]}$ To decipher the unconventional AIE phenomenon, the restriction of intramolecular rotation was proved as the mechanism based on solid evidence. ${ }^{[2]}$ With the development of various AIEgens, the mechanism of AIE phenomenon is further enriched by restriction of intramolecular vibration and thus it is unified to restriction of intramolecular motion (RIM) as a more general mechanism. ${ }^{[3]}$ Compared with traditional organic luminophores, AIEgens enjoy many advantages such as high brightness and excellent photostability in aggregated state and

Z. Song, W. Zhang, M. Jiang,

Dr. H. H. Y. Sung, Dr. R. T. K. Kwok,

Prof. I. D. Williams,

Prof. B. Z. Tang

Department of Chemistry

Institute for Advanced Study

Division of Biomedical Engineering

Division of Life Science

State Key Laboratory of Molecular Neuroscience

and Institute of Molecular

Functional Materials

The Hong Kong University

of Science and Technology

Clear Water Bay, Kowloon, Hong Kong, China

E-mail: tangbenz@ust.hk

H. Nie, Prof. B. Z. Tang

Guangdong Innovative Research Team

SCUT-HKUST Joint Research Laboratory

State Key Laboratory of Luminescent

Materials and Devices

South China University of Technology

Guangzhou 510640, China

Prof. B. Liu

Department of Chemical and

Biomolecular Engineering

National University of Singapore

4 Engineering Drive 4, Singapore 117585, Singapore

E-mail: cheliub@nus.edu.sg

Prof. B. Z. Tang

HKUST Shenzhen Research Institute

No. 9 Yuexing First RD

South Area, Hi-Tech Park

Nanshan, Shenzhen 518057, China

DOI: $10.1002 / \mathrm{adfm} .201503788$

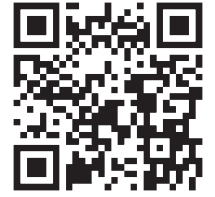
low fluorescence in molecularly dissolved state. ${ }^{[4]}$ For example, in cellular imaging, some fluorescent dyes have been designed to accumulate in specific organelles. For traditional dyes, if too many molecules accumulate in a specific site, the concentration quenching effect will come into play which leads to fluorescence quenching. ${ }^{[5]}$ However, AIEgens will enjoy such an aggregation process to yield enhanced fluorescence with high photobleaching resistance, which is highly desirable for biological applications.

To date, numerous AIEgens have been developed based on different core structures such as tetraphenylethene (TPE), ${ }^{[6]}$ hexaphenylsilole (HPS), ${ }^{[7]}$ distyrylanthracene (DSA), ${ }^{[8]}$ boron diiminates, ${ }^{[9]}$ and tetraphenylpyrazine $(\mathrm{TPP})^{[10]}$ (Scheme 1). However, most of these core structures emit in short wavelength region, normally blue or green. To redshift their emission, researchers have made great synthetic efforts on modification of molecular structures. In our previous work, an AIE-active red emitter was prepared successfully but the synthetic procedure was complicated and laborious. ${ }^{[4 \mathrm{~d}]}$ To the best of our knowledge, so far there is no single AIE system whose emission could be tuned from blue to red by simply altering electron acceptors (donors).

Recently our group reported a new class of AIEgen based on TPP, which introduces hetero atoms and donor-acceptor (D-A) interaction into the molecular design. ${ }^{[10]}$ Although they possess good thermal-, photo-, and chemostabilities, their emission is only tunable from 390 to $460 \mathrm{~nm}$. Similar to the structure of pyrazine, imidazole is a typical five-membered N-heterocycle compound, whose derivatives are widely used as dyes, ${ }^{[11]}$ catalysts, ${ }^{[12]}$ drugs, 


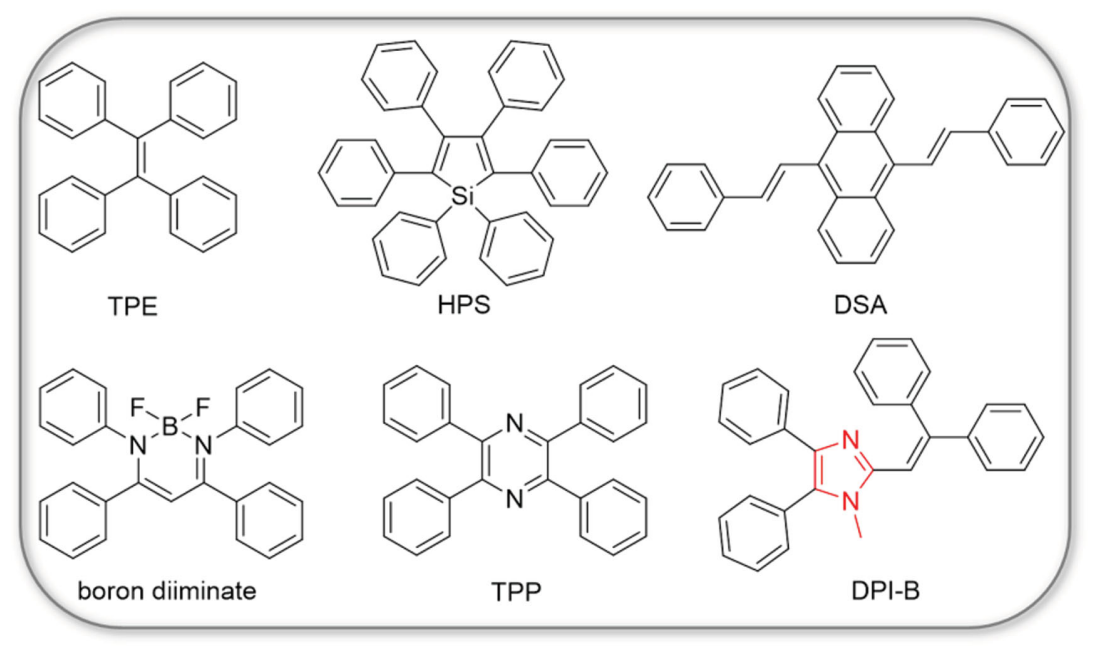

Scheme 1. Molecular structures of TPE, HPS, DSA, boron diiminates, TPP, and DPI-B. organelle-specific fluorescent probes. Moreover, inspired by many commercial antifungal drugs containing imidazole moiety, we screened the imidazole derivatives and one shows potent antifungal activity. This successful study reveals the close relationship between structure and property, thus promoting the development of novel AIEgens.

\section{Results and Discussion}

\subsection{Synthesis and Characterization of the Imidazole Derivatives}

All diphenylimidazole (DPI) derivatives, including DPI-B, DPI-A, DPI-BP, and DPI-In, were synthesized through onestep coupling reaction from 1-methyl4,5-diphenyl-1 $H$-imidazole-2-carbaldehyde and ionic liquids. ${ }^{[12 a, 13]}$ Meanwhile the 2-position of imidazole is active due to inductive effects from the two adjacent nitrogen atoms, which facilitates further functionalization. On the other hand, imidazole moiety could act as either electron donors or acceptors and D-A interaction could be facilely introduced into the molecular design. In consideration of its convenient functionalization and flexible electronic effect, imidazole derivatives with rotatable parts may be a good candidate to construct AIEgens meeting the requirements proposed above.

In this contribution, we report the synthesis of several imidazole-based analogues, investigation of their optical properties including AIE characteristics, and exploration of their biological applications. This new family of AIEgens can be synthesized through less than four steps and one representative structure (DPI-B) is shown in Scheme 1. It is noteworthy that the emission color of the imidazole-based AIEgens varies from blue to deep red by selecting appropriate acceptors. The synthetic accessability of these AIEgens enables us to design
(DPI-CHO) under different conditions (Scheme 2). DPI-CHO was prepared by methylation of commercially available 4,5-diphenylimidazole, followed by formylation reaction under basic condition (Scheme S1, Supporting Information). DPI-B was prepared by McMurry coupling reaction between DPI-CHO and benzophenone. DPI-A, DPI-BP, and DPI-In were obtained through condensation reactions between DPI$\mathrm{CHO}$ and 2-phenylacetonitrile, 4-benzyl-1-methylpyridium iodide, 1,2,3,3-tetramethyl-3 $\mathrm{H}$-indolium iodide, respectively. DPI-BP and DPI-In further underwent anion exchange to replace iodide with hexafluorophosphate, which avoids the fluorescence quenching effect of heavy atoms. The four imidazole derivatives were purified by column chromatography followed by recrystallization with slow evaporation of solvents at ambient temperature. All the compounds were characterized by ${ }^{1} \mathrm{H}$ NMR, ${ }^{13} \mathrm{C}$ NMR, and high resolution mass spectrometry (Figures S1-S15, Supporting Information), which confirmed their correct structures.

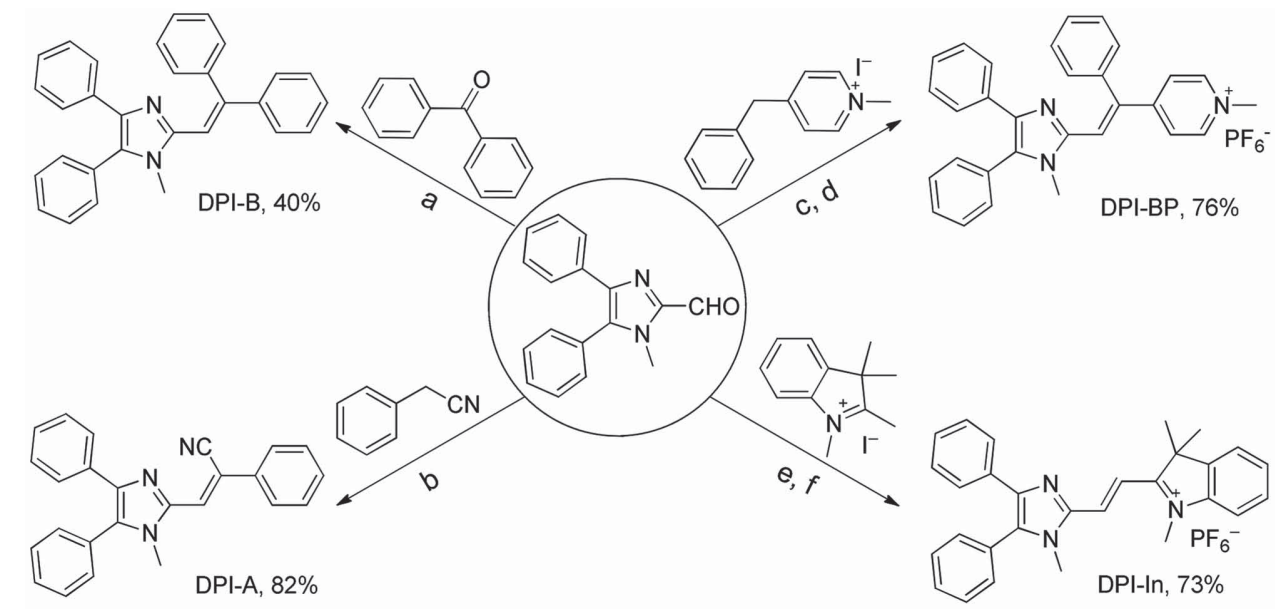

Scheme 2. Synthetic routes to DPI-B, DPI-A, DPI-BP, and DPI-In. Reagents and conditions: a) $\mathrm{TiCl}_{4}, \mathrm{Zn}, \mathrm{THF}$, reflux, 10-12 h; b) NaOH, EtOH, room temperature, 4-6 h; c) piperidine, EtOH, reflux, 10-12 h; d) $\mathrm{KPF}_{6}$, acetone, room temperature, $4 \mathrm{~h}$; e) EtOH, reflux, 10-12 h; and f) $\mathrm{KPF}_{6}$, acetone, room temperature, $4 \mathrm{~h}$. 


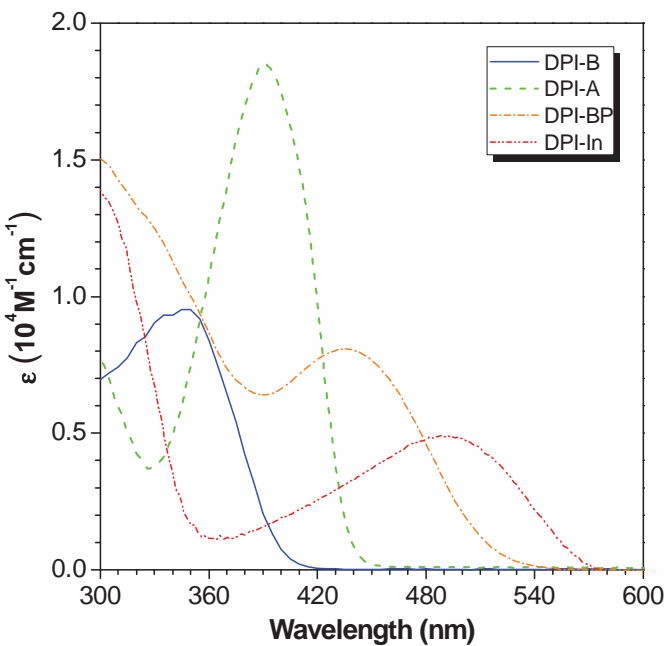

Figure 1. Absorption spectra of DPI-B, DPI-A, DPI-BP, and DPI-In in dimethylsulfoxide (DMSO) solution.

\subsection{Optical Properties}

After confirming the structures of imidazole derivatives, we first investigated their optical properties. As a core structure in the series of compounds, imidazole may serve as either an electron withdrawing group or an electron donating group, dependent on the moieties conjugated to it. For DPI-B, imidazole acts as a weak electron withdrawing group since the substituents on it are phenyl rings and diphenylvinyl group. From DPI-A to DPI-BP and DPI-In, electron withdrawing groups, namely cyano, pyridium, and indolium, are introduced to the vinyl group conjugated with imidazole thus the imidazole core changes its role to serve as an electron donor. As presented in the absorption spectra of the four molecules (Figure 1), DPI-B, DPI-A, DPI-BP, and DPI-In show the longest wavelength absorption peaked at 350, 390, 435, and $492 \mathrm{~nm}$, respectively. The gradual bathochromic shift from DPI-B to DPI-In should be ascribed to the increasing electron affinity (phenyl $<$ cyano group $<$ pyridium $<$ indolium), which will strengthen D-A effect and thereby reduce the energy gaps between excited states and ground states.

In the design of these imidazole derivatives, there are rotatable parts including vinyl group and different aromatic rings around the imidazole core. According to the RIM mechanism of AIE, these compounds may have AIE signature, implied by the composition of cores and rotors. We first checked DPI-B in tetrahydrofuran (THF)-water mixtures. As shown in Figure 2A, when the water fraction increases from $0 \%$ to $70 \%$, the photoluminescence (PL) spectra of DPI-B keep at a low level and show no obvious change. When the water fraction is above $90 \%$, the PL intensity of DPI-B in THF-water mixtures is boosted significantly to yield approximately sevenfold enhancement. DPI-A and DPI-BP also show the similar phenomenon (Figures S16 and S17, Supporting Information). The relative PL intensities $\left(I / I_{0}\right)$ of DPI-B, DPI-A, and DPI-BP at different fractions of poor solvents are plotted in Figure 2B. It is obvious that all of the three compounds are AIE-active. Unfortunately, for DPI-In, due to its strong intramolecular charge transfer (ICT) character, the emission becomes weaker in THF/water or DMSO/water solvent systems with increasing water fractions, which indicates that the ICT process plays a dominant role when water fractions increase and thus AIE effect could not be displayed by monitoring the PL spectra changes. ${ }^{[14]}$ However, we find that the quantum yields of DPI-In in solution state and solid state are 0.008 and 0.049 , respectively, evidently suggesting that DPI-In is indeed AIE-active. As shown in Table 1, the quantum yields of DPI-B, DPI-A, and DPI-BP in solid state are also much higher than those in solution state (10-25-fold), which further confirm their AIE characteristics. Other photophysical parameters are also summarized in Table 1. It is worth mentioning that the absorption peaks of the four compounds in film state only redshift slightly (less than $10 \mathrm{~nm}$ ) compared with those in solution state. This phenomenon implies that short-range intermolecular interactions such as $\pi-\pi$ interaction and dimer formation are lacking in the solid state. Meanwhile,
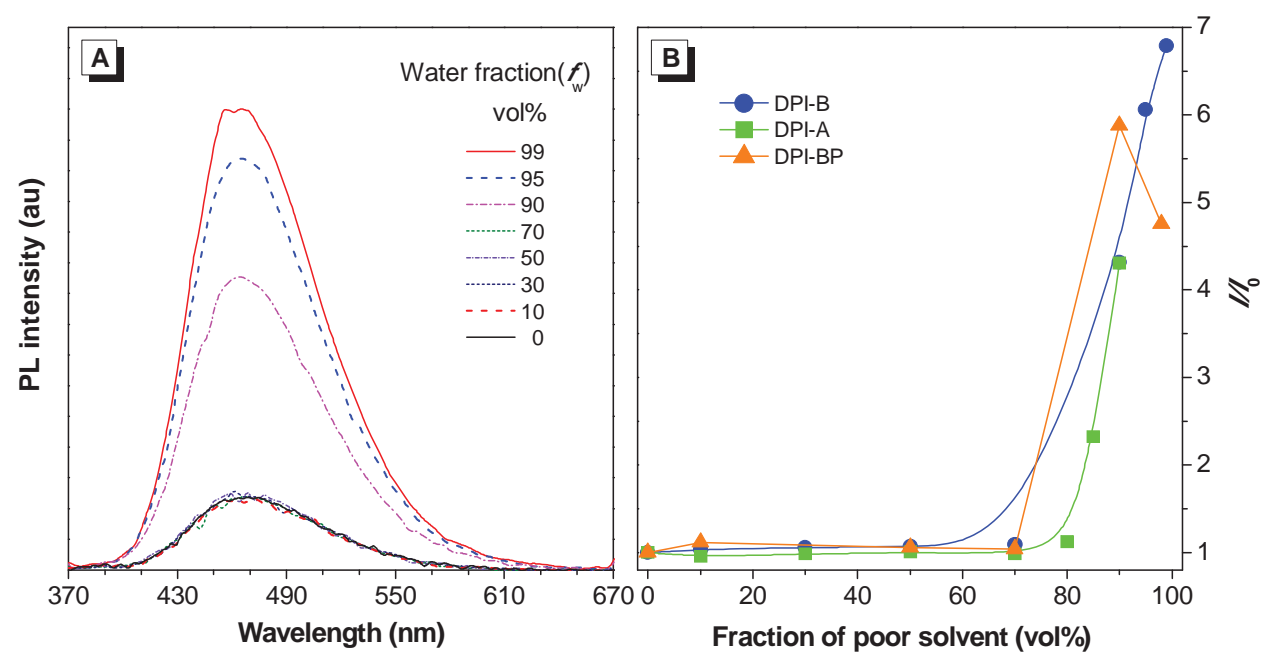

Figure 2. A) PL spectra of DPI-B in THF/water mixtures with different fractions $\left(f_{w}\right)$. B) Variation in relative PL intensity $\left(I / I_{0}\right)$ of $D P I-B$, DPI-A, and $\mathrm{DPI}-\mathrm{BP}$ with different fractions of poor solvent. Concentration: $10 \times 10^{-6} \mathrm{M}$; poor solvent: water for DPI-B, DPI-A and hexane for DPI-BP. 
Table 1. Photophysical properties of DPI-B, DPI-A, DPI-BP, and DPI-In in solution and solid state.

\begin{tabular}{|c|c|c|c|c|c|c|c|}
\hline \multirow[t]{2}{*}{ Compound } & \multicolumn{3}{|c|}{ Solution ${ }^{\text {a) }}$} & \multicolumn{3}{|c|}{ Solid } & \multirow[t]{2}{*}{$E_{\mathrm{g}}^{\mathrm{b})}[\mathrm{eV}]$} \\
\hline & $\lambda_{\text {abs }}[\mathrm{nm}]$ & $\lambda_{\mathrm{em}}[\mathrm{nm}]$ & $\Phi_{\mathrm{f}}^{\mathrm{c})}$ & $\lambda_{\mathrm{abs}}{ }^{\mathrm{d})}[\mathrm{nm}]$ & $\lambda_{\mathrm{em}}{ }^{\mathrm{e})}[\mathrm{nm}]$ & $\Phi_{\mathrm{f}}^{\mathrm{c})}$ & \\
\hline DPI-B & 350 & 470 & 0.010 & 352 & 475 & 0.253 & 3.11 \\
\hline DPI-A & 390 & 474 & 0.008 & 400 & 500 & 0.152 & 2.85 \\
\hline DPI-BP & 435 & 595 & 0.009 & 436 & 616 & 0.142 & 2.41 \\
\hline DPI-In & 492 & 614 & 0.008 & 501 & 642 & 0.049 & 2.19 \\
\hline
\end{tabular}

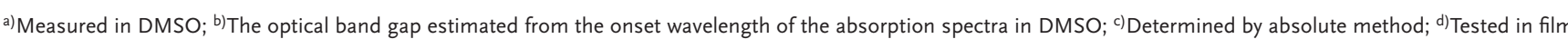
state; e) Excitation wavelength: $325 \mathrm{~nm}$ (DPI-B), $405 \mathrm{~nm}$ (DPI-A), and $470 \mathrm{~nm}$ (DPI-BP and DPI-In).

the photographs (Figure 3 ) of the powders of DPI-B, DPI-A, DPI-BP, and DPI-In under $365 \mathrm{~nm}$ UV illumination clearly show the prominent advantages of the imidazole-based AIEgens: tunable emission colors from blue to red, which agree with their corresponding emission maxima at 475, 500, 616, and $642 \mathrm{~nm}$.

To better understand the optical behavior of these imidazolebased AIEgens, density functional theory calculations were performed using the B3LYP/6-31G(d,p) basis set. As shown in Figure 4, the highest occupied molecular orbitals (HOMOs) are fully delocalized over the entire molecular backbone, whereas the lowest unoccupied molecular orbitals (LUMOs) are localized on the imidazole core and the electron-withdrawing groups, exhibiting the D-A structural features. From DPI-B to DPI-In, the energy levels of HOMOs and LUMOs are both lowered. Particularly, with the increase of electron affinity of acceptors, LUMOs are dramatically decreased from $-1.33 \mathrm{eV}$ for DPI-B to $-5.79 \mathrm{eV}$ for DPI-In, faster than the decrease of HOMOs. Therefore, the energy gaps become smaller. On the other hand, the optical band gaps estimated from the onset wavelength of the absorption spectra show a decreasing trend from $3.11 \mathrm{eV}$ for DPI-B to $2.19 \mathrm{eV}$ for DPI-In (Table 1). All these agree fairly well with the gradually redshifted absorption maxima for DPI-A, DPI-BP, and DPI-In.

\subsection{Crystal Structures}

To gain insight into the AIE nature of the imidazole derivatives as well as the variation of their emission colors, we have obtained the single crystal structures to disclose their molecular conformation and packing modes. The single crystal structures of DPI-B, DPI-A, DPI-BP, and DPI-In are shown in Figure 5. In general, all four imidazole-based AIEgens adopt nonplanar conformations and the twisted angles between the phenyl rings at 4, 5-position of imidazole and the core vary from $12.5^{\circ}$ to $82.5^{\circ}$. Moreover, the packing modes show that each compound stacks in the form of head-to-tail and no strong intermolecular interaction is observed except for some weak interactions such as $\mathrm{C}-\mathrm{H} \cdots \mathrm{C}$ and $\mathrm{C}-\mathrm{H} \cdots \mathrm{N}$ (Figure S18, Supporting Information). These results clearly demonstrate that typical $\pi-\pi$ interaction, which often brings about quenching effect in concentrated condition, does not exist between adjacent molecules of the four compounds. We further looked into the twisted angles between the imidazole core and the vinyl group. It is interesting to find that the values are decreased from $43.9^{\circ}$ to $1.1^{\circ}$ with the redshifted emission. The smaller twisted angles indicate more planar conformation of vinylimidazole moiety and better conjugation of the whole molecule, which is perfectly consistent with their emission maxima. All these data, including stator-rotor structures, twisted conformations, and crystal packing modes, should be responsible for the AIE feature of the four compounds.

\subsection{Mitochondria-Specific Imaging}

Recent studies have revealed that pyridium, indolium, and other positively charged molecules are able to target mitochondria efficiently. ${ }^{[15]}$ We then envision that the four imidazole-based molecules, especially DPI-BP and DPI-In may also be used for mitochondria staining. We started with the examination of the cytotoxicity of imidazole-based AIEgens by 3-(4,5-dimethyl-2-thiazolyl)-2,5-diphenyl-2 $\mathrm{H}$-tetrazolium bromide (MTT) assay. As summarized in Figure S19 (Supporting Information), DPI-B, DPI-BP, and DPI-In show low cytotoxicity while DPI-A shows some cytotoxicity at high concentrations, which is probably due to the liberation of trace amount of cyanide from DPI-A. ${ }^{[16]}$ Therefore we chose $5 \times 10^{-6} \mathrm{M}$ as the AIEgen concentration for the subsequent experiments.

Fluorescent images of HeLa cells stained with DPI-B, DPI-A, DPI-BP, and DPI-In are displayed in Figure 6. The results show that the reticulum structures of mitochondria are clearly lightened up by the yellow fluorescence from DPI-BP with a high

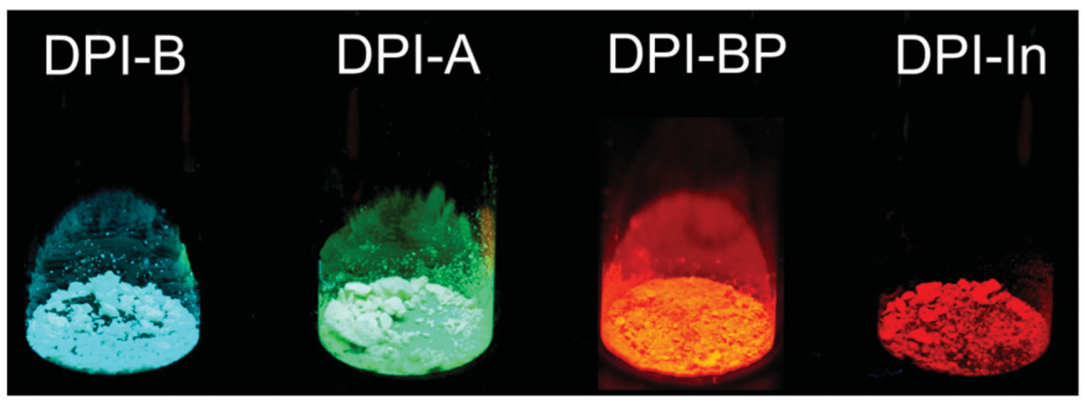

Figure 3. Photographs of powders of DPI-B, DPI-A, DPI-BP, and DPI-In taken under $365 \mathrm{~nm}$ UV illumination. 


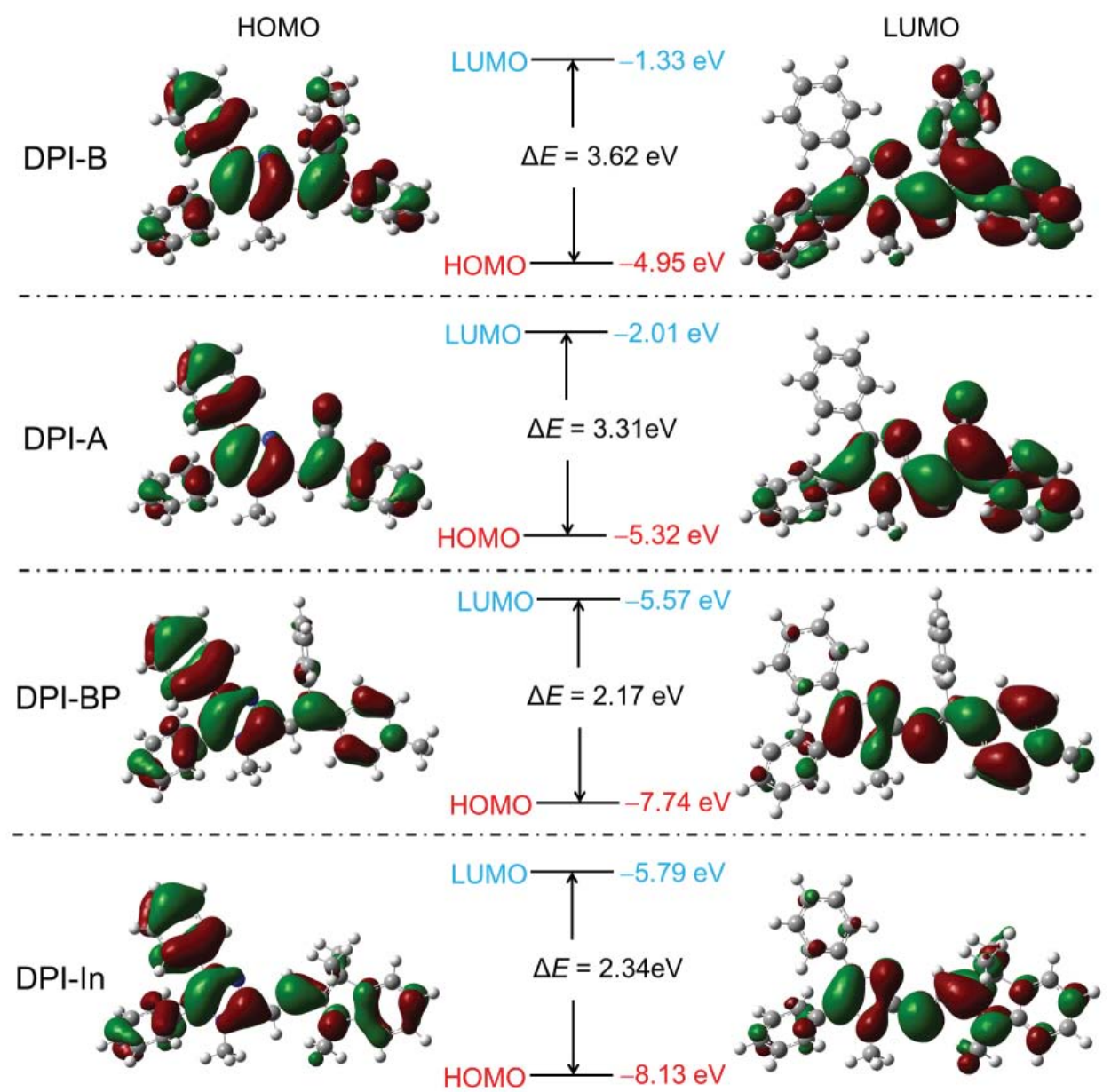

Figure 4. Molecular orbital amplitude plots of HOMO and LUMO energy levels of DPI-B, DPI-A, DPI-BP, and DPI-In calculated by using the B3LYP/6$31 \mathrm{G}(\mathrm{d}, \mathrm{p})$ basis set.

imaging contrast. Regarding to DPI-B, DPI-A, and DPI-In, they could also accumulate in mitochondria since the reticulum structures show brighter emission than cytoplasm, but the signal-to-background ratio is not as high as DPI-BP. Furthermore, the four compounds were used to costain HeLa cells with mito-tracker red (MTR), a commercially available mitochondria imaging agent. As presented in Figure 7 and Figure S20 (Supporting Information), fluorescent signals of MTR and DPI-BP collected from different channels are well matched with an overlapping coefficient of 0.92 . The other three compounds, DPI-B, DPI-A, and DPI-In, show inferior performance in terms of specificity to mitochondria, with an overlapping coefficient of $0.66,0.74$, and 0.76 , respectively. These results suggest that DPI-BP indeed selectively stains mitochondria and pyridium moiety plays a more important role in the specific targeting.

\subsection{Antifungal Activity}

Another application we further explored was antifungal capability of the imidazole derivatives. In consideration of biosafety, the nonpathogenic fungus, yeast, is selected as a representative species for the antifungal study. DPI-B, DPI-A, DPI-BP, and DPI-In were first screened by monitoring the optical density (OD) change of yeasts incubated with the four imidazole derivatives in aqueous phase. As illustrated in Figure 8, DPI-B, DPI-A, and DPI-BP exhibit no obvious inhibition effect on the proliferation of yeasts while DPI-In inhibits the growth of yeasts potently. To verify the inhibition specificity of DPI-In to fungi, the same experiments were conducted on bacteria (e.g., Escherichia coli) and the results demonstrate that DPI-In is totally ineffective to bacteria (Figure S21, Supporting Information). Combining with the MTT results mentioned above, we can preliminarily conclude that DPI-In only exerts inhibition effect on fungi, which could be a potential antifungal drug. To further investigate the antifungal activity of DPI-In, we compare it with the commercially available drug, miconazole and the results are presented in Figure 9A. It is clear that $5 \times 10^{-6} \mathrm{M}$ of DPI-In and $10 \times 10^{-6} \mathrm{M}$ of miconazole exhibit similar inhibition effect on proliferation of yeasts while the inhibition efficiency of $5 \times 10^{-6} \mathrm{M}$ miconazole is not conspicuous, indicating DPI-In has a lower concentration threshold of inhibition effect. Moreover, the antifungal activity of DPI-In, miconazole and DPI-BP were also evaluated by disc diffusion method (Figure 9B), ${ }^{[17]}$ which shows the much larger inhibition zone around DPI-In and demonstrates that DPI-In is a more effective antifungal agent than miconazole and other newly 

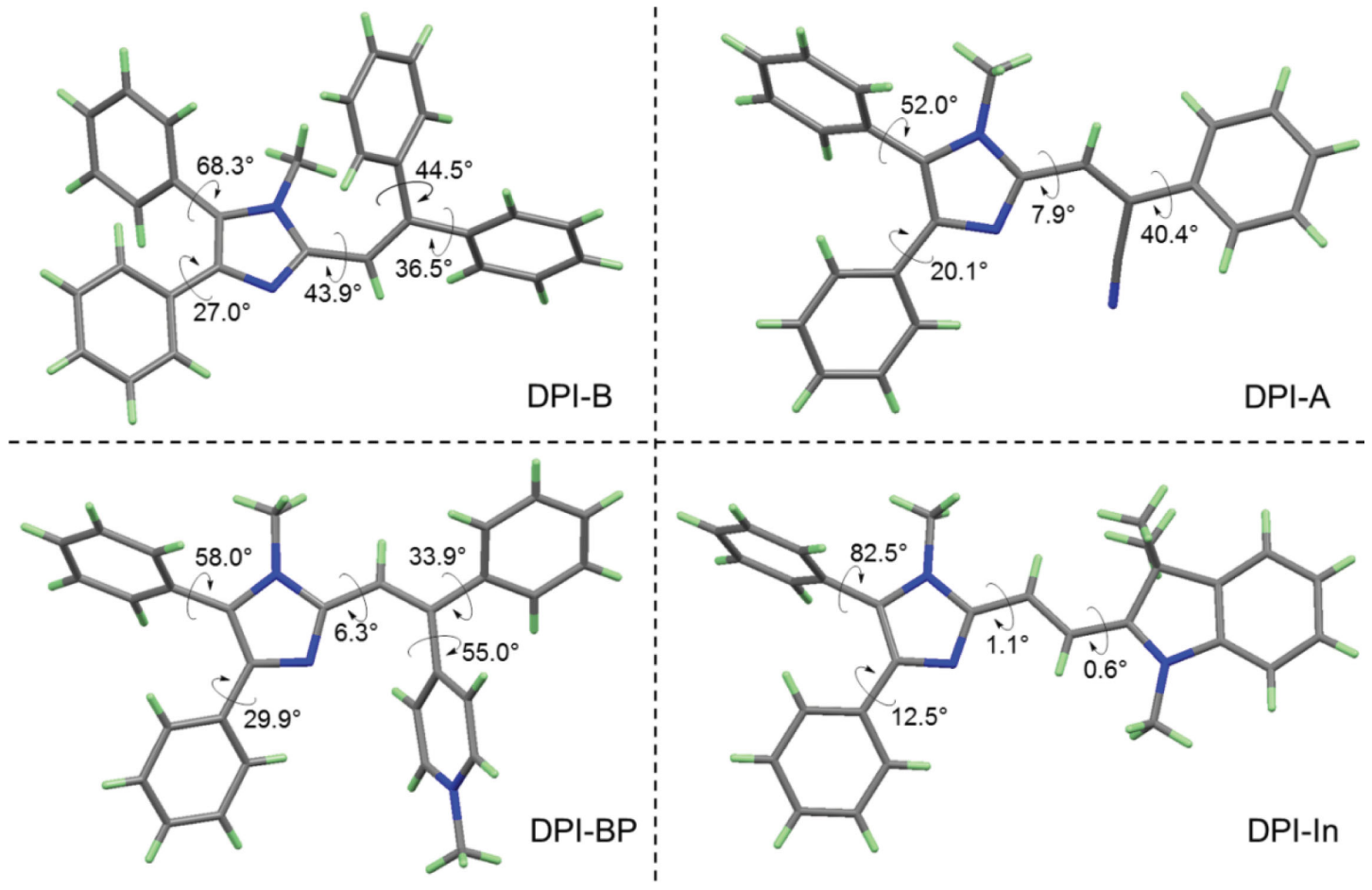

Figure 5. Single-crystal structures of DPI-B, DPI-A, DPI-BP, and DPI-In. Carbon, hydrogen, and nitrogen atoms are shown in gray, green, and blue, respectively.

developed imidazole derivatives under the same condition. The time-dependent and concentration-dependent OD changes of yeasts incubated with DPI-In are shown in Figure S22A,B (Supporting Information), respectively. According to the curve plotted by concentration-dependent OD changes incubated
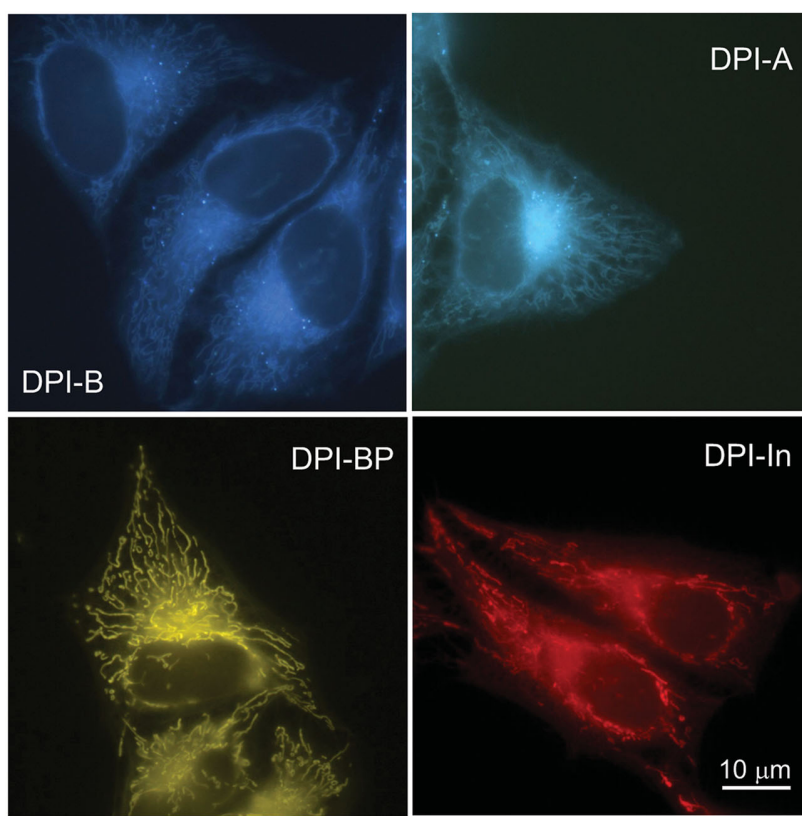

Figure 6. Fluorescent images of HeLa cells stained with DPI-B, DPI-A, $\mathrm{DPI}-\mathrm{BP}$, and DPI-In for $15 \mathrm{~min}$. Concentration: $5 \times 10^{-6} \mathrm{M}$; Excitation: $330-385 \mathrm{~nm}$ (DPI-B and DPI-A), 400-440 nm (DPI-BP), and 460-490 nm (DPI-In). All figures share the same scale bar. with DPI-In, the $\mathrm{IC}_{50}$ of DPI-In for inhibition of yeast growth is estimated to be $4 \times 10^{-6} \mathrm{M}$, which is similar to most commercial antifungal drugs. ${ }^{[18]}$ As a new compound, DPI-In has been verified to show the interesting property but detailed mechanism awaits further investigation. In addition, DPI-In could not only serve as an inhibitor of fungi but also stain live bacteria and fungi, helping researchers to visualize them more clearly (Figure S23, Supporting Information).

\section{Conclusion}

We designed a group of imidazole derivatives with facile synthesis and wide color tunability. Based on the imidazole core, the emission colors can be tuned from blue to deep red, when different acceptors (cyano, pyridium, and indolium) are conjugated to the core. The single crystal structures reveal that the smaller twisted angles between the imidazole and the vinyl group lead to a more planar conformation as well as a more redshifted emission. With an imidazole core as the stator and aromatic rings as the rotors, all the compounds show typical AIE characteristics, which could be mechanistically supported by their twisted structures and absence of $\pi-\pi$ interaction in crystal packing. We further explored their biological applications including mitochondria-specific imaging and antifungal activity. DPI-BP performs the best in targeting mitochondria, giving high-resolution images of the specific organelle due to the presence of pyridium moiety. DPI-In is screened to be the most potent antifungal agent among these molecules, which shows better performance than the commercially available antifungal drug of miconazole. This successful study should 

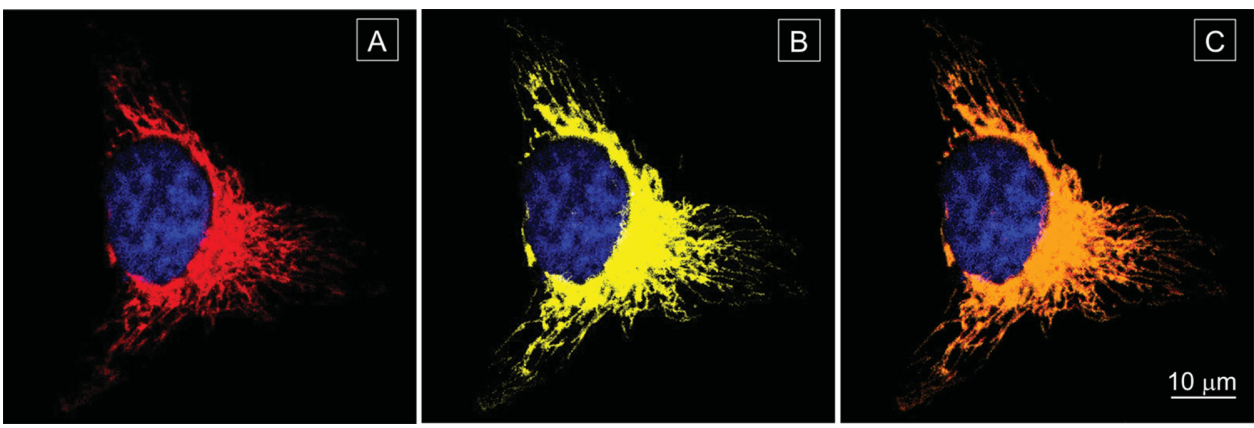

Figure 7. CLSM images of HeLa cells stained with A) mito-tracker red $\left(50 \times 10^{-9} \mathrm{M}\right)$ and $\left.\mathrm{B}\right) \mathrm{DPI}-\mathrm{BP}$ for 15 min. C) Merged image of $(\mathrm{A})$ and $(\mathrm{B})$. Excitation wavelength: $405 \mathrm{~nm}$ (DPI-BP) and $560 \mathrm{~nm}$ (mito-tracker red). Nuclei were live-stained with Hoechst 33342.

intrigue the development of novel AIEgens and extend more high-tech applications of AIE materials.

\section{Experimental Section}

Materials and Instrumentation: All chemicals and reagents were commercially available and used as received without further purification. THF was distilled from sodium benzophenone ketyl under nitrogen immediately prior to use. Ethanol (EtOH) was dried in anhydrous $\mathrm{MgSO}_{4}$ and filtered before use. 4,5-Diphenylimidazole, methyl iodide, benzophenone, 4-benzylpyridine, 2-phenylacetonitrile, zinc powder, and 2,3,3-trimethylindolenine were purchased from Aldrich. Titanium (IV) chloride was purchased from Merck Co. and superdry $\mathrm{N}, \mathrm{N}$-dimethyl formamide (DMF) was obtained from J\&K Co. Minimum essential medium (MEM), fetal bovine serum (FBS), penicillin-streptomycin solution, and MTR FM were purchased from Invitrogen. Hoechst 33342, 3-(4,5-dimethyl-2-thiazolyl)-2,5diphenyltetrazolium bromide (MTT), and miconazole were purchased from Aldrich. Yeast (YPH500), E. coli, and HeLa cell were obtained from the American Type Culture Collection. ${ }^{1} \mathrm{H}$ and ${ }^{13} \mathrm{C}$ NMR spectra were measured on a Bruker ARX $400 \mathrm{NMR}$ spectrometer using $\mathrm{CDCl}_{3}$ and $\mathrm{CD}_{2} \mathrm{Cl}_{2}$ as solvents and tetramethylsilane (TMS; $\delta=0 \mathrm{ppm}$ ) was chosen as internal reference. Melting points were measured with digital melting point apparatus (Barnstead/Electrothermal). UV spectra were measured on a Biochrom Libra S80PC double beams spectrometer. PL spectra were recorded on a Perkin-Elmer LS 55 spectrofluorometer.

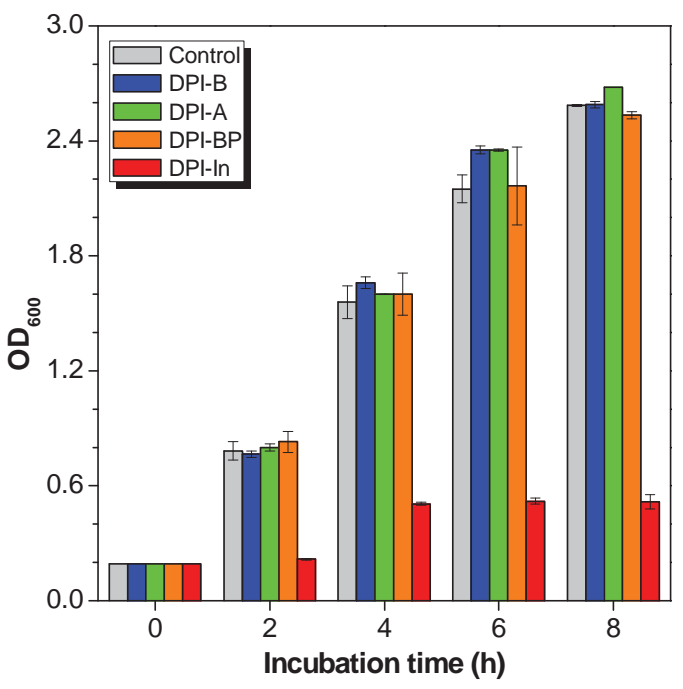

Figure 8. Optical density change of yeast incubated in different imidazole derivatives with different incubation time. Concentration: $5 \times 10^{-6} \mathrm{M}$.
High-resolution mass spectra (HRMS) were recorded on a GCT Premier CAB 048 mass spectrometer operating in matrix-assisted laser desorption/ionization-time of flight (MALDI-TOF) mode. The fluorescence quantum efficiencies were measured using a calibrated integrating sphere (Labsphere). Fluorescent images were taken on a fluorescent microscope (BX41 Microscope) and confocal lasing scanning microscopic (CLSM) images were obtained on a confocal microscope (Zeiss Laser Scanning Confocal Microscope; LSM7 DUO) using ZEN 2009 software (Carl Zeiss).

Synthesis of DPI-CHO: DPI-CHO was synthesized according to literature. ${ }^{[19]}$ Briefly, 4,5-diphenylimidazole was first methylated by methyl iodide to generate 1-methyl-4,5-diphenylimidazole. To a solution of 1-methyl-4,5-diphenylimidazole $(4 \mathrm{mmol}, 937 \mathrm{mg}$ ) in dry THF, $n$-butyllithium ( $4.4 \mathrm{mmol}, 2.76 \mathrm{~mL}$ of a $1.6 \mathrm{M}$ solution in cyclohexanes) was added dropwise under nitrogen at $-78^{\circ} \mathrm{C}$. After $1 \mathrm{~h}$ DMF $(1.7 \mathrm{~mL}$, $22 \mathrm{mmol}$ ) was added dropwise. The mixture was kept at $-78{ }^{\circ} \mathrm{C}$ for another $1 \mathrm{~h}$ and then allowed to warm to ambient temperature. After stirred further for $2 \mathrm{~h}$, the reaction was quenched with saturated ammonium chloride $(50 \mathrm{~mL})$ and the resulting solution was extracted with ethyl acetate for three times. The organic layer was combined and washed with brine $(50 \mathrm{~mL})$, dried $\left(\mathrm{MgSO}_{4}\right)$, and concentrated under reduced pressure. The crude product was purified by silica gel chromatography with hexane/ethyl acetate $(4: 1)$ as eluent to afford the desired product $(776 \mathrm{mg}, 2.96 \mathrm{mmol}$ ) as white to light yellow solid. Yield $=74 \%$. ${ }^{1} \mathrm{H}$ NMR $\left(400 \mathrm{MHz}, \mathrm{CDCl}_{3}\right): \delta(\mathrm{TMS}, \mathrm{ppm}) 9.92(\mathrm{~s}, 1 \mathrm{H})$, 7.51-7.45 (m, 5H), 7.35-7.34 (m, 2H), 7.25-7.23 (m, 3H), $3.84(\mathrm{~s}, 3 \mathrm{H})$. ${ }^{13} \mathrm{C}$ NMR $\left(100 \mathrm{MHz}, \mathrm{CDCl}_{3}\right): \delta$ (TMS, ppm) 181.73, 142.42, 140.68, $135.09,132.63,129.83,129.00,128.64,127.72,126.79,126.48,32.28$. HRMS (MALDI-TOF), $\mathrm{m} / \mathrm{z}$ calcd. for $\mathrm{C}_{17} \mathrm{H}_{14} \mathrm{~N}_{2} \mathrm{O}$ : 262.1106; found $262.1101\left(\mathrm{M}^{+}\right)$

Synthesis of DPI-B: To a solution of DPI-CHO $(0.5 \mathrm{mmol}, 131 \mathrm{mg})$, benzophenone (1.5 mmol, $273.3 \mathrm{mg})$, and zinc powder $(4.8 \mathrm{mmol}, 313.7 \mathrm{mg})$ in dry $\mathrm{THF}, \mathrm{TiCl}_{4}(2.4 \mathrm{mmol}, 0.263 \mathrm{~mL})$ was added dropwise at $-78^{\circ} \mathrm{C}$. Then the reaction mixture was warmed to ambient temperature and heated to reflux for 10-12 h. After cooled down, the reaction mixture was filtered and extracted with dichloromethane for three times. The organic layer was combined, dried $\left(\mathrm{MgSO}_{4}\right)$, and concentrated under reduced pressure. The crude product was purified by silica gel chromatography with hexane/ethyl acetate $(9: 1)$ as eluent to afford the desired product (82 mg, $0.2 \mathrm{mmol}$ ) as white solid. Yield $=40 \%$. m.p. $=184.8-185.5^{\circ} \mathrm{C}$. ${ }^{1} \mathrm{H}$ NMR $\left(400 \mathrm{MHz}, \mathrm{CD}_{2} \mathrm{Cl}_{2}\right): \delta$ (TMS, ppm) 7.44-7.33 (m, $\left.13 \mathrm{H}\right)$, 7.27-7.22 (m, 4H), 7.15-7.10 (m, 3H), $6.86(\mathrm{~s}, 1 \mathrm{H}), 2.98(\mathrm{~s}, 3 \mathrm{H})$. ${ }^{13} \mathrm{C}$ NMR $\left(100 \mathrm{MHz}, \mathrm{CD}_{2} \mathrm{Cl}_{2}\right): \delta$ (TMS, ppm) $146.85,144.20,141.81$, $139.42,136.59,134.32,130.62,130.09,129.67,128.63,128.24,127.79$, $127.52,127.36,127.25,127.14,125.77,125.36,115.12,30.90$. HRMS (MALDI-TOF), $m / z$ calcd. for $\mathrm{C}_{30} \mathrm{H}_{24} \mathrm{~N}_{2}$ : 412.1939; found 412.1933 (M+).

Synthesis of DPI-A: To a solution of DPI-CHO $(0.4 \mathrm{mmol}, 105 \mathrm{mg})$ and 2-phenylacetonitrile $(0.44 \mathrm{mmol}, 51.5 \mathrm{mg})$ in dry $\mathrm{EtOH}$, a solution of $\mathrm{NaOH}(50 \mathrm{mg}$ ) in $\mathrm{EtOH}$ was added dropwise at room temperature. The reaction mixture was further stirred for $4-6 \mathrm{~h}$ and the precipitates were filtered followed by washing with ethanol and hexane. After dried in vacuum, the yellow solid was obtained as the target product 


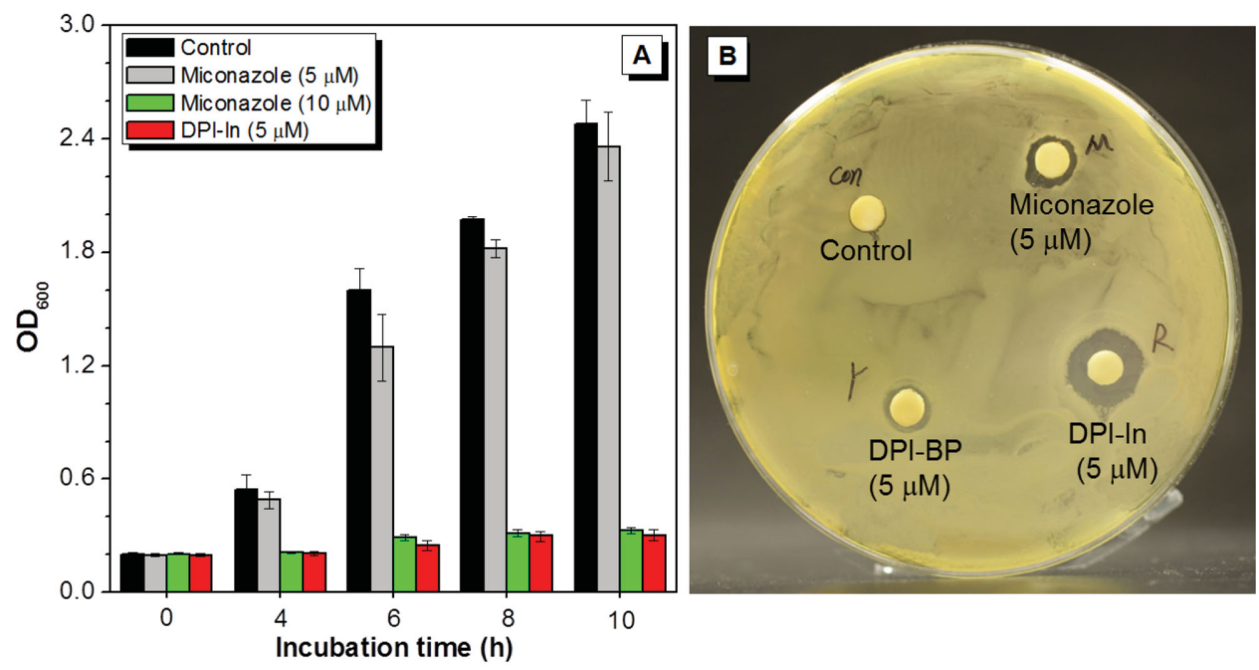

Figure 9. A) Optical density change of yeast incubated with different antifungal agents with different incubation time. B) Antifungal activity of different antifungal agents evaluated by disc diffusion method.

$(118 \mathrm{mg}, 0.33 \mathrm{mmol})$. Yield $=82 \% . \mathrm{m} . \mathrm{p} .=194.6-195.8{ }^{\circ} \mathrm{C} .{ }^{1} \mathrm{H}$ NMR $\left(400 \mathrm{MHz}, \mathrm{CDCl}_{3}\right): \delta$ (TMS, ppm) 7.75-7.73 (d, 2H), 7.61-7.60 (d, 2H), 7.50-7.35 (m, 8H), $7.32(\mathrm{~s}, 1 \mathrm{H}), 7.23-7.17(\mathrm{~m}, 3 \mathrm{H}), 3.54(\mathrm{~s}, 3 \mathrm{H}) .{ }^{13} \mathrm{C}$ NMR $\left(100 \mathrm{MHz}, \mathrm{CDCl}_{3}\right): \delta$ (TMS, ppm) 139.32, 133.86, 133.23, 131.33, $130.13,129.58,128.76,128.59,128.49,127.60,126.35,126.31,125.55$ $123.86,116.88,112.25,30.48$. HRMS (MALDI-TOF), $\mathrm{m} / \mathrm{z}$ calcd. for $\mathrm{C}_{25} \mathrm{H}_{19} \mathrm{~N}_{3}$ : 361.1579 ; found $361.1569\left(\mathrm{M}^{+}\right)$.

Synthesis of DPI-BP: 4-Benzyl-7-methylpyridium iodide was first prepared through methylation of 4-benzylpyridine. A solution of DPI-CHO $(0.3 \mathrm{mmol}, 79 \mathrm{mg})$ and 4-benzyl-1-methylpyridium iodide $(0.3 \mathrm{mmol}, 93.4 \mathrm{mg})$ in dry EtOH was refluxed for 10-12 $\mathrm{h}$ in the presence of one or two drops of piperidine as catalyst. After cooled down, the reaction mixture was concentrated under reduced pressure and purified by silica gel chromatography with dichloromethane/MeOH (98:2-95:5) as eluent to afford the product $(126 \mathrm{mg}, 0.23 \mathrm{mmol})$ as orange solid. Then the counter ion of the product was changed from $\mathrm{I}^{-}$to $\mathrm{PF}_{6}{ }^{-}$after stirring it with $\mathrm{KPF}_{6}$ in acetone for $4 \mathrm{~h}$. Yield: $76 \%$. m.p. $=222.8-224.6{ }^{\circ} \mathrm{C} .{ }^{1} \mathrm{H}$ NMR $\left(400 \mathrm{MHz}\right.$, DMSO- $\left.d_{6}\right): \delta$ (TMS, ppm) 9.07-9.06 (d, 2H), 8.13-8.11 (d, 2H), 7.54-7.53 (m, 3H), 7.52-7.43 $(\mathrm{m}, 5 \mathrm{H}), 7.39(\mathrm{~s}, 1 \mathrm{H}), 7.36-7.34(\mathrm{~m}, 2 \mathrm{H}), 7.14-7.11(\mathrm{~m}, 3 \mathrm{H}), 6.91-6.89$ (d, $2 \mathrm{H}), 4.46(\mathrm{~s}, 3 \mathrm{H}), 3.58(\mathrm{~s}, 3 \mathrm{H}) .{ }^{13} \mathrm{C}$ NMR $\left(100 \mathrm{MHz}\right.$, DMSO-d $\left.d_{6}\right): \delta$ (TMS, ppm) 158.44, 144.86, 142.33, 138.53, 137.48, 136.81, 133.92, $130.40,130.09,129.93,129.08,129.02,128.90,128.66,128.60,128.00$, $127.13,126.18,125.46,115.53,47.10,30.82$. HRMS (MALDI-TOF), $\mathrm{m} / \mathrm{z}$ calcd. for $\left(\mathrm{C}_{30} \mathrm{H}_{26} \mathrm{~N}_{3}\right)^{+}$: 428.2121 ; found $429.2232(\mathrm{M}+\mathrm{H})^{+}$.

Synthesis of DPI-In: The synthetic procedure of DPI-In was similar to that of DPI-BP. Yield: $73 \%$. m.p. $=198.6-199.7^{\circ} \mathrm{C} .{ }^{1} \mathrm{H}$ NMR $(400 \mathrm{MHz}$, DMSO- $\left.d_{6}\right): \delta$ (TMS, ppm) 8.10-8.06 (d, $\left.1 \mathrm{H}\right), 7.96-7.95(\mathrm{~m}, 1 \mathrm{H})$ 7.90-7.88 (m, 1H), 7.77-7.74 (d, 1H), 7.65-7.59 (m, 5H), 7.49-7.45 $(\mathrm{m}, 4 \mathrm{H}), 7.32-7.28(\mathrm{~m}, 3 \mathrm{H}), 4.13(\mathrm{~s}, 3 \mathrm{H}), 3.78(\mathrm{~s}, 3 \mathrm{H}), 1.82(\mathrm{~s}, 6 \mathrm{H})$. ${ }^{13} \mathrm{C}$ NMR $\left(100 \mathrm{MHz}\right.$, DMSO- $\left.d_{6}\right): \delta$ (TMS, ppm) 180.33, 143.27, 142.63 , $141.82,141.75,135.85,134.97,133.02,130.25,129.58,129.18,129.09$, $128.86,128.80,128.19,127.47,126.65,122.72,114.91,111.75,51.81$, $34.14,31.52,25.33$. HRMS (MALDI-TOF), $\mathrm{m} / \mathrm{z}$ calcd. for $\left(\mathrm{C}_{29} \mathrm{H}_{28} \mathrm{~N}_{3}\right)^{+}$. 418.2278; found $418.2289\left(\mathrm{M}^{+}\right)$.

Cell Culture: The HeLa cells were cultured in MEM containing $10 \%$ FBS, $100 \mathrm{U} \mathrm{mL}^{-1}$ penicillin, and $100 \mu \mathrm{g} \mathrm{mL}^{-1}$ streptomycin in a $5 \% \mathrm{CO}_{2}$ humidity incubator at $37^{\circ} \mathrm{C}$.

Cell Imaging: HeLa cells were grown in a $35 \mathrm{~mm}$ Petri dish with a coverslip at $37^{\circ} \mathrm{C}$. The living cells were stained with the four imidazolebased molecules $\left(5 \times 10^{-3} \mathrm{M}\right)$ for $15 \mathrm{~min}$. The cells were washed for three times with phosphate-buffered saline (PBS) buffer and imaged under fluorescent microscope (BX41 Microscope) using different combination of excitation and emission filters for each dye. For the DPI-B and
DPI-A, the excitation filter $=330-385 \mathrm{~nm}$, dichroic mirror $=400 \mathrm{~nm}$, and the emission filter $=420 \mathrm{~nm}$ long pass. For DPI-BP, the excitation filter $=400-440 \mathrm{~nm}$, dichroic mirror $=455 \mathrm{~nm}$, and the emission filter $=465 \mathrm{~nm}$ long pass. For DPI-In, the excitation filter $=460-490 \mathrm{~nm}$, dichroic mirror $=505 \mathrm{~nm}$, and the emission filter $=515 \mathrm{~nm}$ long pass. For costaining experiments, HeLa cells were stained with DPI-BP $\left(5 \times 10^{-6} \mathrm{M}\right)$ and MTR $\left(50 \times 10^{-9} \mathrm{M}\right)$ for $15 \mathrm{~min}$. After washed twice with PBS, the cells were stained with Hoechst 33342 for 5 min, followed by further washing with PBS. The cells were imaged by CLSM. DPI-BP was excited at $458 \mathrm{~nm}$ and the fluorescence was collected at 500-600 nm. MTR was excited at $560 \mathrm{~nm}$ and the fluorescence was collected at $600-750 \mathrm{~nm}$. Hoechst 33342 was excited at $405 \mathrm{~nm}$ and the fluorescence was collected at $420-500 \mathrm{~nm}$. For the CLSM images stained by the other three dyes with MTR, DPI-B, DPI-A, DPI-In, and MTR were excited at 405, 405, 488, and $560 \mathrm{~nm}$, respectively.

Cell Viability Evaluated by MTT Assay: In brief, $100 \mu \mathrm{L}$ of $\mathrm{HeLa}$ cell was seeded per well in a 96-well plate at the density of $5 \times 10^{4}$ cell $\mathrm{mL}^{-1}$. After overnight culture, the medium was removed and the adherent cells were washed twice with PBS. Various dyes with a series of doses were added to the 96-well plate. After incubation for $12 \mathrm{~h}, 10 \mu \mathrm{L}$ of freshly prepared MTT solution $\left(5 \mathrm{mg} \mathrm{mL}^{-1}\right)$ in PBS was added into each well. After incubation for $4 \mathrm{~h}, 100 \mu \mathrm{L}$ of solubilization solution (containing $10 \%$ sodium dodecyl sulfate and $0.01 \mathrm{M} \mathrm{HCl}$ ) was added to each well to dissolve the purple crystals. After $8 \mathrm{~h}$ incubation, the plate was gently shaken for $1 \mathrm{~min}$ at room temperature and the absorbance of individual well at 595 well was monitored using a plate reader. Each of the experiments was performed at least three times.

Antimicrobial Studies: Prior to antimicrobial tests, the YPH500 were maintained on yeast extract peptone dextrose (YPD) culture plate (1\% yeast extract, $2 \%$ peptone, $2 \%$ glucose, $2 \%$ agar), E. coli was cultured in Luria broth medium. For the antimicrobial studies, a single colony of microbe on the solid culture medium was transferred to $10 \mathrm{~mL}$ of liquid medium in tube and cultured under vigorous shaking at $30{ }^{\circ} \mathrm{C}$. The concentration of microbe was diluted to an optical density at $600 \mathrm{~nm}$ (OD600) of nearly 0.2. Different dyes or drugs with various predetermined concentrations were added to the individual tube. The same amount of pure DMSO was also added to the tube for negative control. After designed time intervals, the concentrations of microbe were determined by measuring OD600. The tests were repeated for three times to ensure reliability. For the disc diffusion method, The YPH500 was painted on the surface of YPD culture plate and the discs which had been impregnated with different dyes $\left(2 \times 10^{-3} \mathrm{M}\right.$ in DMSO) were then placed on the surface of solid culture medium. The plate was incubated at $37^{\circ} \mathrm{C}$ for $12 \mathrm{~h}$. 
CCDC 1414518, 1414678, 1414519, and 1414520 contain the supplementary crystallographic data for this paper. These data can be obtained free of charge from The Cambridge Crystallographic Data Centre via www.ccdc.cam.ac.uk/data_request/cif.

\section{Supporting Information}

Supporting Information is available from the Wiley Online Library or from the author.

\section{Acknowledgements}

Z.S. and W.Z. contributed equally to this work. This work was partially supported by the National Basic Research Program of China (973 Program; 2013CB834701), the Research Grants Council of Hong Kong (16301614, 16305015, and N HKUST604/14), Innovation and Technology Commission (ITCPD/17-9), the University Grants Committee of Hong Kong (AoE/P-03/08), Singapore National Research Foundation (R279-000-444-281), and the Singapore Ministry of Defense (R279-000340-232). B.Z.T. is also grateful for the support from the Guangdong Innovative Research Team Program of China (201101C0105067115). The authors would also like to express thanks to Dr. Zikai He, Dr. Yilin Zhang, and Miss Chen Gui for their help in this project.

Received: September 7, 2015

Revised: September 30, 2015 Published online: December 17, 2015

[1] a) J. Luo, Z. Xie, J. W. Y. Lam, L. Cheng, B. Z. Tang, H. Chen, C. Qiu, H. S. Kwok, X. Zhan, Y. Liu, D. Zhu, Chem. Commun. 2001, 381, 1740; b) Y. Hong, J. W. Y. Lam, B. Z. Tang, Chem. Soc. Rev. 2011, 40, 5361; c) J. Liang, B. Z. Tang, B. Liu, Chem. Soc. Rev. 2015, 44, 2798.

[2] J. Chen, C. C. W. Law, J. W. Y. Lam, Y. Dong, S. M. F. Lo, I. D. Williams, D. Zhu, B. Z. Tang, Chem. Mater. 2003, 15, 1535.

[3] J. Mei, Y. Hong, J. W. Y. Lam, A. Qin, Y. Tang, B. Z. Tang, Adv. Mater. 2014, 26, 5429.

[4] a) D. Ding, K. Li, B. Liu, B. Z. Tang, Acc. Chem. Res. 2013, 46, 2441; b) M. Wang, G. Zhang, D. Zhang, D. Zhu, B. Z. Tang, J. Mater. Chem. 2010, 20, 1858; c) Y. Liu, Y. Yu, J. W. Y. Lam, Y. Hong, M. Faisal, W. Z. Yuan, B. Z. Tang, Chem. Eur. J. 2010, 16, 8433; d) W. Qin, D. Ding, J. Liu, W. Z. Yuan, Y. Hu, B. Liu, B. Z. Tang, Adv. Funct. Mater. 2012, 22, 771.

[5] a) F. L. Tansi, R. Rüger, M. Rabenhold, F. Steiniger, A. Fahr, W. a. Kaiser, I. Hilger, Small 2013, 9, 3659; b) J.-P. Francoia, R. Pascal, L. Vial, Chem. Commun. 2015, 51, 1953; c) Y. Gao, Y. Kuang, X. Du, J. Zhou, P. Chandran, F. Horkay, B. Xu, Langmuir 2013, 29, 15191.
[6] a) Y. Dong, J. W. Y. Lam, A. Qin, J. Liu, Z. Li, B. Z. Tang, J. Sun, H. S. Kwok, Appl. Phys. Lett. 2007, 91, 011111; b) X. Wang, J. Hu, G. Zhang, S. Liu, J. Am. Chem. Soc. 2014, 136, 9890

[7] a) X. Fan, J. Sun, F. Wang, Z. Chu, P. Wang, Y. Dong, R. Hu, B. Z. Tang, D. Zou, Chem. Commun. 2008, 381, 2989; b) T. Zhang, Y. Jiang, Y. Niu, D. Wang, Q. Peng, Z. Shuai, J. Phys. Chem. A 2014, 118, 9094

[8] a) J. He, B. Xu, F. Chen, H. Xia, K. Li, L. Ye, W. Tian, J. Phys. Chem. C 2009, 113, 9892; b) J. Zhang, B. Xu, J. Chen, S. Ma, Y. Dong, L. Wang, B. Li, L. Ye, W. Tian, Adv. Mater. 2014, 26, 739; c) X. Li, K. Ma, S. Zhu, S. Yao, Z. Liu, B. Xu, B. Yang, W. Tian, Anal. Chem. 2014, 86, 298.

[9] R. Yoshii, A. Hirose, K. Tanaka, Y. Chujo, J. Am. Chem. Soc. 2014, 136, 18131.

[10] M. Chen, L. Li, H. Nie, J. Tong, L. Yan, B. Xu, J. Z. Sun, W. Tian, Z. Zhao, A. Qin, B. Z. Tang, Chem. Sci. 2015, 6, 1932.

[11] a) P. Molina, A. Tárraga, F. Otón, Org. Biomol. Chem. 2012, 10, 1711; b) R. Li, S. Xiao, Y. Li, Q. Lin, R. Zhang, J. Zhao, C. Yang, K. Zou, D. Li, T. Yi, Chem. Sci. 2014, 5, 3922; c) W. Li, W. Lin, J. Wang, X. Guan, Org. Lett. 2013, 15, 1768.

[12] a) J. Dupont, R. F. De Souza, P. A. Z. Suarez, Chem. Rev. 2002, 102, 3667; b) R. Martínez, I. M. Pastor, M. Yus, Eur. J. Org. Chem. 2014, $2014,872$.

[13] a) L. Zhang, X. Peng, G. L. V Damu, R. Geng, C. Zhou, Med. Res. Rev. 2013, 34, 340; b) M. A. Ghannoum, L. B. Rice, Clin. Microbiol. Rev. 1999, 12, 501; c) Z. Si, L. Qiu, H. Dong, F. Gu, Y. Li, F. Yan, ACS Appl. Mater. Interfaces 2014, 6, 4346.

[14] R. Hu, E. Lager, A. Aguilar-Aguilar, J. Liu, J. W. Y. Lam, H. H. Y. Sung, I. D. Williams, Y. Zhong, K. S. Wong, E. Peña-Cabrera, B. Z. Tang, J. Phys. Chem. C 2009, 113, 15845.

[15] a) S. W. Perry, J. P. Norman, J. Barbieri, E. B. Brown, H. A. Gelbard, Biotechniques 2011, 50, 98; b) A. Mathur, Y. Hong, B. K. Kemp, A. Alvarez, J. D. Erusalimsky, Cardiovasc. Res. 2000, 46, 126; c) Y. Chen, C. Zhu, Z. Yang, J. Chen, Y. He, Y. Jiao, W. He, L. Qiu, J. Cen, Z. Guo, Angew. Chem. Int. Ed. 2013, 52, 1688; d) L. Yuan, L. Wang, B. K. Agrawalla, S.-J. Park, H. Zhu, B. Sivaraman, J. Peng, Q.-H. Xu, Y.-T. Chang, J. Am. Chem. Soc. 2015, 137, 5930; e) L. Zhang, W. Liu, X. Huang, G. Zhang, X. Wang, Z. Wang, D. Zhang, X. Jiang, Analyst 2015, 140, 5849; f) N. Zhao, M. Li, Y. Yan, J. W. Y. Lam, Y. L. Zhang, Y. S. Zhao, K. S. Wong, B. Z. Tang, J. Mater. Chem. C 2013, 1, 4640.

[16] H. Tanii, K. Hashimoto, Arch. Toxicol. 1984, 55, 47.

[17] A. Kathiravan, K. Sundaravel, M. Jaccob, G. Dhinagaran, A. Rameshkumar, D. A. Ananth, T. Sivasudha, J. Phys. Chem. B 2014, 118, 13573.

[18] a) W. Zhang, Y. Ramamoorthy, T. Kilicarslan, H. Nolte, R. F. Tyndale, E. M. Sellers, Drug Metab. Dispos. 2002, 30, 314; b) T. Niwa, T. Shiraga, A. Takagi, Biol. Pharm. Bull. 2005, 28, 1805.

[19] M. J. Plater, P. Barnes, L. K. McDonald, S. Wallace, N. Archer, T. Gelbrich, P. N. Horton, M. B. Hursthouse, Org. Biomol. Chem. 2009, 7, 1633. 